\title{
CONHECIMENTO E USO DA CONTRACEPÇÃO DE EMERGÊNCIA NA ADOLESCÊNCIA: CONTRIBUIÇÕES PARA A ENFERMAGEM
}

\author{
Milena de Freitas Rodrigues ${ }^{1}$, Dulcilene Pereira Jardim²
}

\begin{abstract}
RESUMO: Trata-se de estudo descritivo-exploratório com o objetivo de identificar o conhecimento e o uso da contracepção de emergência entre adolescentes. A coleta de dados deu-se por meio de questionário respondidos por 271 adolescentes de uma escola pública de São Paulo em 2010. Os resultados revelam que 87,8\% das adolescentes conhecem a pílula e a forma de utilização, 28,8\% já usaram a pílula e o uso médio por adolescente foi de três vezes e de forma correta. A contracepção de emergência não foi usada por todas as adolescentes que tiveram a sexarca ou demais relações sexuais sem proteção, o que representa uma lacuna entre o conhecimento e o uso dessa modalidade de contracepção. Cabe ao enfermeiro, como educador atuante em unidades básicas de saúde, escolas e outros espaços sociais, desenvolver estratégias para alcançar esta população e contribuir para o conhecimento, a prevenção da gravidez indesejada e promoção do autocuidado.
\end{abstract}

PALAVRAS-CHAVE: Adolescente; Anticoncepcionais pós-coito; Educação em saúde; Enfermagem.

\section{KNOWLEDGE AND USE OF EMERGENCY CONRACEPTION IN ADOLESCENCE: CONTRIBUTIONS FOR NURSING}

ABSTRACT: This descriptive-exploratory study aimed to identify the knowledge and use of emergency contraception among adolescents. Data collection was through a questionnaire responded to by 271 adolescents from a state school in São Paulo in 2010. The results show that $87.8 \%$ of the adolescents know the morning-after pill and how to use it, $28.8 \%$ had already used the morning-after pill, and the average use per adolescent was three times - and used correctly. Emergency contraception was not used by all the adolescents who had initiated sexual activity or who had sex or other sexual relations without protection, which represents a gap between knowledge and use of this form of contraception. It falls to the nurse, as an educator working in primary health care centers, schools and other social spaces, to develop strategies for reaching this population and contributing to its knowledge, to the prevention of unwanted pregnancy, and to the promotion of self-care.

KEYWORDS: Adolescent; Post-coital contraception; Health education; Nursing.

\section{CONOCIMIENTO Y USO DE LA ANTICONCEPCIÓN DE EMERGENCIA EN LA ADOLESCENCIA: CONTRIBUCIONES PARA LA ENFERMERÍA}

RESUMEN: Este es un estudio descriptivo-exploratorio cuyo objetivo fue identificar el conocimiento y el uso de la anticoncepción de emergencia entre adolescentes. Los datos fueron obtenidos por medio de cuestionarios contestados por 271 adolescentes de una escuela pública de São Paulo en 2010. Los resultados revelan que 87,8\% de las adolescentes conocen la píldora y su forma de utilización, 28,8\% ya usaron la píldora y el uso medio por adolescente fue de tres veces y de forma correcta. La anticoncepción de emergencia no fue usada por todas adolescentes que tuvieron la sexarca o demás relaciones sexuais sin protección, lo que representa un hueco entre el conocimiento y el uso de esa modalidad de anticoncepción. Es responsabilidad del enfermero, como educador actuante en unidades básicas de salud, escuelas y otros espacios sociales, desarrollar estrategias para alcanzar esta población y contribuir para el conocimiento, la prevención de la gravidez indeseada y la promoción del autocuidado.

PALABRAS CLAVE: Adolescente; Contracepción pos relación sexual; Educación en salud; Enfermería.

${ }^{1}$ Acadêmica de Enfermagem pela Universidade de Santo Amaro.
${ }^{2}$ Enfermeira. Mestre em Ciências. Professora do Curso de Graduação em Enfermagem da Universidade de Santo Amaro. 


\section{INTRODUÇÃO}

A adolescência, fase que compreende entre 10 a 19 anos de idade, é um período permeado de alterações físicas e psíquicas e paralelamente a este processo pode dar-se início a vida sexual ${ }^{(1)}$. Esta iniciação pode tornar o adolescente vulnerável à situações indesejadas, como a ocorrência de gravidez, contaminação por Doenças Sexualmente Transmissíveis (DST), entre outras, que repercutirão em seu futuro pessoal e profissional e/ou da qualidade de vida ${ }^{(2)}$. Para prevenção destes riscos, vê-se a importância do uso de métodos contraceptivos como um direito que possibilita, cada vez mais, o ser humano ao exercício da sexualidade desvinculado da procriação ${ }^{(3)}$.

Entre os métodos, os preservativos masculino e/ou feminino ganham destaque como recurso que atende à função de proteção contra gravidez indesejada e as DST/ AIDS simultaneamente. Porém, ainda é comum a resistência ao uso do preservativo ${ }^{(4)}$ entre os adolescentes, grupo no qual seu uso aumentou mas, ainda não é utilizado por todos os adolescentes nem em todas as relações sexuais ${ }^{(5)}$.

Na ocorrência de uma relação sexual desprotegida, o Contraceptivo de Emergência (CE) ou Pílula do Dia Seguinte torna-se uma valiosa opção ${ }^{(6)}$. A CE é reconhecida como alternativa contraceptiva em situações emergenciais após uma relação sexual desprotegida, falha anticoncepcional presumida ou nos casos de violência sexual ${ }^{(7)}$. No Brasil, a CE faz parte da Política Nacional de Saúde da Mulher do Ministério da Saúde e cujo foco é garantir a autonomia feminina no planejamento familiar. Consta também no Marco Teórico Referencial da Saúde Sexual e Reprodutiva do Adolescente e do Marco Legal Saúde - um direito de adolescentes. Esta política estabeleceu como definitiva a condição não abortiva da CE, validando seu uso em qualquer etapa da vida reprodutiva considerando a antecipação da puberdade e do início da atividade sexual ${ }^{(2)}$.

Atualmente, a CE é vendida em farmácias, sem necessidade de prescrição médica, além de ser distribuída nas Unidades Básicas de Saúde, inclusive para adolescentes. Porém, a facilidade no acesso tem despertado receios por parte de profissionais de saúde que atuam com adolescentes. As preocupações são relativas à possibilidade do uso abusivo do método e suas implicações, além do receio de que ocorra o abandono do uso do preservativo, em prol da CE, devido ao entendimento, parcial ou equivocado, sobre a utilização do método somente em situações de emergência, e a não prevenção das DST/Aids ${ }^{(6)}$. Dados da Pesquisa Nacional de Demografia e Saúde da Criança e da
Mulher de 2006 revelou que a CE é o terceiro método mais usado entre adolescentes de 15 a 19 anos (10,4\%), ficando atrás apenas do uso da camisinha masculina $(50,3 \%)$ e da pílula $(36,8 \%)^{(6,8)}$.

Para a correta compreensão sobre a CE faz-se necessária a orientação oferecida por profissionais de saúde, entre eles o enfermeiro, atuando como educador em saúde para o público adolescente ${ }^{(9)} \mathrm{em}$ diversos cenários sociais, seja em ambiente escolar ou nas unidades de saúde ${ }^{(10)}$. Estas, podem propiciar espaços de diálogo entre adolescentes, professores, profissionais de saúde e família, para a construção de uma resposta social, com vistas à superação das relações de vulnerabilidade às DST/Aids, assim como da gravidez precoce ${ }^{(11)}$.

Diante desse cenário, a presente pesquisa teve por objetivo identificar o conhecimento e o uso da CE entre adolescentes do sexo feminino.

\section{MÉTODO}

Trata-se de um estudo descritivo-exploratório realizado em uma Escola Estadual localizada na zona Sul do Município de São Paulo que oferece Ensino Fundamental II (5 à $8^{\mathrm{a}}$ série) e Ensino Médio $\left(1^{\mathrm{o}}\right.$ ao $3^{\circ}$ ano) com um total de 1.500 alunos distribuídos nos períodos matutino, vespertino e noturno. Entre os alunos, aproximadamente 800 são do sexo feminino.

A amostra de 271 adolescentes foi do tipo não probabilística por conveniência, cujos critérios de inclusão foram: ter entre 10 e 19 anos, ser do sexo feminino e matriculadas na escola em questão, que manifestasse interesse em participar do estudo, além de obter a permissão expressa do seu responsável legal. A pesquisa foi aprovada pelo Comitê de Ética em Pesquisa da Universidade de Santo Amaro sob o protocolo n. 074/10.

A coleta de dados deu-se no mês de outubro e novembro de 2010, após a apresentação do estudo por parte do pesquisador, bem como a assinatura do Termo de Consentimento Livre Esclarecido, o qual foi submetido e autorizado pelos pais ou responsáveis. $\mathrm{O}$ instrumento de coleta de dados utilizado foi um questionário semiestruturado com 29 perguntas, abertas e fechadas, divididas em: Parte I - Caracterização sociodemográfica; Parte II - Conhecimento sobre a pílula do dia seguinte e Parte III - Uso da pílula do dia seguinte. $\mathrm{O}$ instrumento foi respondido pelos adolescentes em ambiente escolar nos horários de aula, de acordo com a indicação do coordenador pedagógico e autorização do professor, com duração média de 20 minutos. 


\section{RESULTADOS}

\section{Caracterização sociodemográfica das adolescentes}

A amostra deste estudo foi constituída por 271 adolescentes do sexo feminino com idade entre $10 \mathrm{e}$ 19 anos (média de 14,8 anos), cursando em maior parte o $2^{\circ}$ ano do ensino médio (100/36,9\%).

Em relação ao estado civil apenas $4(1,5 \%)$ das adolescentes eram casadas e $8(2,9 \%)$ mães. A maioria morava com os pais (113/41,7\%), sendo 197 (72,7\%) em casa própria, $65(23,9 \%)$ em casa alugada e 8 $(2,9 \%)$ em casa construída em terreno da prefeitura. A renda familiar desta população concentrava-se entre $\mathrm{R} \$ 501,00$ à $\mathrm{R} \$ 1.000,00(67 / 24,7 \%)$. A renda individual das adolescentes que trabalhavam $(13 / 4,8 \%)$ era de até um salário mínimo ( $\mathrm{R} \$ 510,00), 12$ (4,4\%) recebiam acima de um salário mínimo. Quanto à religião, 154 $(56,8 \%)$ eram católicas, $76(28,0 \%)$ evangélicas, 8 $(2,9 \%)$ espíritas e $16(5,9 \%)$ não possuíam religião.

\section{Aspectos da sexualidade entre as adolescentes}

Entre as adolescentes do estudo, a menarca ocorrera para a totalidade, sendo em média aos 11,7 anos, e a sexarca ocorreu para 132 (48,7\%) e em média aos 13,7 anos. Quando questionadas sobre o uso de contraceptivo na sexarca, $110(40,6 \%)$ referiram ter usado, em sua maioria (99/90,0\%) a camisinha, seguida da pílula (11/10,0\%).

A CE foi utilizada por apenas $3(13,6 \%)$ das 22 adolescentes que não usaram método contraceptivo. Nas relações sexuais seguintes, a pílula foi o método mais utilizado (53/19,5\%); e o uso simultâneo da camisinha e da pílula foi referido por 38 (14,0\%) delas. Cinco (1,8\%) adolescentes referiram a utilização da CE nas relações sexuais subsequentes, nos casos de acidentes com o uso do preservativo masculino ou ainda porque o parceiro se recusou a usá-la.

A gestação foi vivenciada por $13(4,8 \%)$ das adolescentes, aos 15,6 anos em média, ou seja, menos de dois anos após a sexarca neste grupo. Duas $(0,7 \%)$ dessas adolescentes recorreram ao aborto para solucionar $\mathrm{o}$ contexto da gravidez indesejada.

\section{Conhecimento da Contracepção de Emergência entre as adolescentes}

As adolescentes foram questionadas quanto ao conhecimento sobre a CE e $238(87,8 \%)$ referiram conhecer, e atribuíram-no este aos meios de comunicação em massa, entre eles a internet, televisão e revistas (153/56,4\%); os amigos (139/51,3\%); aos pais (86/31,7\%) e aos professores $(67 / 24,7 \%)$ na escola. Os demais resultados relativos ao conhecimento das adolescentes sobre a CE encontram-se na Tabela 1.

Tabela 1 - Distribuição das variáveis referentes ao conhecimento da Contracepção de Emergência entre as 271 adolescentes. São Paulo, 2010

\begin{tabular}{|c|c|c|}
\hline VARIÁVEIS & $\mathbf{N}$ & $\%$ \\
\hline \multicolumn{3}{|c|}{ Quantas pílulas devem ser tomadas } \\
\hline Existe com 1 e com 2 pílulas & 94 & 34,7 \\
\hline Só uma pílula & 83 & 30,6 \\
\hline Só duas pílulas & 80 & 29,5 \\
\hline Não respondeu & 14 & 5,2 \\
\hline \multicolumn{3}{|c|}{$\begin{array}{l}\text { Tempo máximo para tomar a } \mathrm{CE}^{*} \text { após relação } \\
\text { desprotegida }\end{array}$} \\
\hline 24 horas & 145 & 53,5 \\
\hline 72 horas & 109 & 40,2 \\
\hline Pode ser tomar a qualquer hora & 04 & 1,5 \\
\hline Não respondeu & 13 & 4,8 \\
\hline \multicolumn{3}{|c|}{ Intervalo entre a $1^{\mathrm{a}}$ pílula e a $2^{\mathrm{a}}$ pílula } \\
\hline 12 horas & 170 & 62,7 \\
\hline 24 horas & 71 & 26,2 \\
\hline Pode tomar a qualquer hora & 06 & 2,2 \\
\hline Não respondeu & 24 & 8,9 \\
\hline
\end{tabular}

Reações que podem acontecer após o uso da $\mathrm{CE}^{* * *}$ Alteração do ciclo menstrual $\quad 185 \quad 68,2$ Dor de Cabeça $\quad 77 \quad 28,4$

Náuseas e/ou vômitos $\quad 60 \quad 22,1$

$\begin{array}{lll}\text { Seios doloridos } & 37 & 13,6\end{array}$

Dor de barriga $\quad 25 \quad 9,2$

Diarreia $09 \quad 3,3$

A CE* pode ser utilizada continuamente

$\begin{array}{lll}\text { Não } & 247 & 91,2\end{array}$

Sim $\quad 02 \quad 0,7$

$\begin{array}{lll}\text { Não respondeu } & 22 & 8,1\end{array}$

*Contracepção de Emergência. **Questão de múltipla escolha.

Para as adolescentes, o uso da CE não deve ser feito continuamente devido à possibilidade de ser prejudicial à saúde $(77 / 28,4 \%)$, ter o seu efeito contraceptivo diminuído $(49 / 18,1 \%)$, causar alterações no ciclo menstrual $(26 / 9,6 \%)$ ou dificultar futura gravidez $(15 / 5,5 \%)$.

\section{Uso da Contracepção de Emergência entre ado- lescentes}

Das 271 adolescentes deste estudo, somente 78 $(28,8 \%)$ já usaram a CE sendo a primeira utilização, em média, aos 14 anos, com uso do método em média 3 vezes e cuja caracterização encontra-se na Tabela 2 . 
Tabela 2 - Distribuição das variáveis referente aos aspectos da utilização da Contracepção de Emergência entre as 78 adolescentes que já a utilizaram. São Paulo, 2010

\begin{tabular}{lcc}
\hline \multicolumn{1}{c}{ VARIÁVEIS } & N & \% \\
\hline Tempo entre a relação sexual desprotegida e o \\
uso da CE* \\
Antes de 12 horas & 48 & 61,5 \\
De 12 horas à 24 horas & 20 & 25,6 \\
De 24 horas à 72 horas & 02 & 2,5 \\
Não respondeu & 08 & 10,2 \\
\hline Tipo de CE* usada & & \\
Duas pílulas & 58 & 74,3 \\
Uma pílula & 20 & 25,6 \\
Não respondeu & - & - \\
\hline Tempo entre a 1a e a 2a ${ }^{\text {a pílula }}$ & & \\
Uso correto & 44 & 56,4 \\
Uso incorreto & 16 & 20,5 \\
Não respondeu & 18 & 23,0 \\
\hline Presença de sintomas após o uso da CE* & \\
Sim & 48 & 61,5 \\
Não & 30 & 38,4 \\
Não respondeu & - & - \\
\hline Sintomas após uso da CE* ** & & \\
Alteração do ciclo menstrual & 29 & 37,1 \\
Náuseas e/ou vômitos & 15 & 19,2 \\
Dor de cabeça & 11 & 14,1 \\
Dor de barriga & 08 & 10,2 \\
Seios doloridos & 06 & 7,6 \\
Alteração de humor & 01 & 1.2 \\
Inchaço & 01 & 1,2 \\
Não respondeu & - & - \\
\hline * Contracepcão de Emergência. **Questão de múltipla escolha.
\end{tabular}

Também foi questionado às participantes sobre dúvidas acerca da CE e 88 (32,5\%) referiram ter dúvidas no que se refere à real eficácia, sobre a forma correta de sua utilização e quanto à diminuição de sua eficácia com o uso contínuo, bem como, os efeitos desse uso para a saúde.

\section{DISCUSSÃO}

A menarca, que é reconhecida como um indicador de maturação no desenvolvimento da mulher, bem como a sexarca estão ocorrendo cada vez mais precocemente na atualidade, com curto intervalo entre as duas ${ }^{(12-13)}$ expondo as adolescentes às vulnerabilidades do sexo desprotegido como a contaminação por DST/Aids, gravidez precoce e/ou indesejada e o aborto. Neste sentido, recomenda-se o uso combinado da pílula e da camisinha para o grupo, parceria que se mostra muito efetiva como uma garantia em virtude do uso inadequado ou do não uso do preservativo pelos adolescentes.

Mas, na ocorrência de uma relação sexual desprotegida, a CE surge como uma alternativa emergencial, porém, o método não é conhecido por todos os adolescentes ${ }^{(14)}$ fato que pode explicar sua subutilização após a sexarca ou demais relações sexuais desprotegidas entre as participantes deste estudo. $\mathrm{O}$ fato da sexualidade ser abertamente debatida nos meios de comunicação, entre elas, as redes sociais, influencia no conhecimento e no comportamento sexual dos adolescentes ${ }^{(15)}$.

Atualmente são comercializados ou distribuídos dois tipos de CE - dose única ou duas doses que dividem a carga hormonal que compõem a pílula. Nesste caso, o segundo comprimido deve ser tomado 12 horas após o primeiro ${ }^{(6)}$, mas, uma porcentagem expressiva de adolescentes do estudo desconhece esta recomendação que, se descumprida, pode comprometer a eficácia do método. Neste sentido, a proposta da CE em dose única elimina a preocupação em relação ao uso incorreto e a possibilidade do esquecimento da segunda dose ${ }^{(2)}$.

A maior parte das adolescentes referiu que a $\mathrm{CE}$ deve ser tomada até 24 horas após o coito desprotegido, o que é considerado correto se partimos do pressuposto que quanto antes ela for tomada, maior sua eficácia ${ }^{(2)}$. Porém, sabe-se que é preconizado do uso no tempo máximo de 72 horas após a relação sexual desprotegida ${ }^{(6)}$ o que é de conhecimento de grande parte das participantes. A utilização da CE em situações específicas é de conhecimento de quase a totalidade das adolescentes deste estudo, ou seja, deve ser usada nos casos de violência sexual, na ineficácia ou na não utilização de outros métodos anticoncepcionais ${ }^{(6)}$. Contudo, este conceito tem sido motivo de preocupação dos profissionais de saúde que atendem adolescentes, devido à possibilidade do uso abusivo e indisciplinar da CE. Também dos possíveis impactos negativos sobre o comportamento contraceptivo de rotina ${ }^{(7)} \mathrm{com}$ a diminuição ou o abandono do uso do preservativo e exposição às DST/Aids ${ }^{(14)}$.

Este cenário reforça a necessidade de Educação em Saúde Sexual e Reprodutiva para este grupo com a devida capacitação dos profissionais de saúde sobre a $\mathrm{CE}$. Entretanto é um método envolto em mitos, como o fato de ser uma pílula abortiva ${ }^{(6)}$. As adolescentes deste estudo relacionam o uso rotineiro ou abusivo da $\mathrm{CE}$ a prejuízos na sua eficácia, é sabido que o método é pouco eficaz se utilizado repetidamente em curtos intervalos ${ }^{(2,14)}$.

Ressalta-se a necessidade de acompanhar os desdobramentos na saúde sexual e reprodutiva das adolescentes 
consumidoras de CE, orientando-as sobre a sua correta utilização e possibilidade de surgimento de efeitos colaterais $^{(7)}$ como cefaleia, náuseas e vômitos. Também, da ocorrência de vômito nas primeiras duas horas após a ingestão do comprimido e, neste caso, da necessidade de repetir a mesma dosagem ${ }^{(2)}$.

$\mathrm{O}$ uso da CE pode ser feito em qualquer idade mas, entre as adolescentes, o número de usuárias vem crescendo por ser um grupo que está mais exposto à gravidez inde$\operatorname{sejada}^{(7)}$, no entanto, esse método não previne as doenças, fazendo das adolescentes uma população de grande risco para as DST/Aids com destaque para a incidência de infecção pelo Vírus do Papiloma Humano, principal agente causador do câncer de colo uterino associado à iniciação sexual precoce e multiplicidade de parceiros sexuais, característicos desta fase, entre outros fatores ${ }^{(16)}$. É fundamental, portanto, que a assistência à adolescência tenha enfoque de prevenção, incluindo-se a CE, e que leve em consideração as características e singularidades dos adolescentes. Fica claro que seu uso adequado vai ao encontro de seu imediatismo, das constantes mudanças de pensar e sentir, colocando-se, portanto, como opção relevante de prevenção da gravidez indesejada ${ }^{(2)}$.

Para facilitar o acesso e o uso, a CE deve ser parte das ações de prevenção das Unidades Básicas de Saúde e Unidades de Saúde da Família. Respeitando, deste modo, os direitos dos adolescentes à assistência sexual e reprodutiva garantidos pelo Estatuto da Criança e do Adolescente que podem ser realizadas independentemente da autorização da família ou responsável ${ }^{(2)}$. Para tanto, é dever do profissional de saúde, como o enfermeiro, oferecer todas as alternativas de informação de forma clara, fácil, acessível, independente de qualquer circunstância, respeitando e zelando pela privacidade da adolescente e, cabe ao profissional aproveitar o momento da prescrição do contraceptivo de emergência para iniciar a orientação sexual de forma ampla, reforçando a necessidade de adesão à dupla proteção(2).

O desconhecimento sobre as características específicas dos adolescentes e a desvalorização das percepções e significados construídos sobre a saúde e meios terapêuticos distanciam as ações de enfermagem das necessidades e das práticas individuais de cuidados. Deste modo, a ssitência a assistência qualificada em termos de eficácia e resolutividade dos problemas que atingem a saúde dos adolescentes ${ }^{(10)}$. Portanto, há necessidade de capacitação dos enfermeiros ${ }^{(1)}$ pois, ainda que tenha sido reconhecida a importância da dimensão educativa como indissociável à prática de enfermagem em qualquer campo de atuação, ainda há carência da formação pedagógica. Na graduação este tem pouco ou nenhum contato com a reflexão teórica e a discussão sobre os modelos de educação em saúde, com revisão mais aprofundada dos conteúdos e adequada reflexão sobre o assunto ${ }^{(17)}$.

\section{CONCLUSÃO}

Este estudo possibilitou averiguar que a maior parte das adolescentes participantes neste estudo demonstra ter conhecimento sobre a CE nos dois formatos disponíveis, sobre a sua correta utilização em relação ao tempo transcorrido pós-coito desprotegido, bem como quanto ao intervalo entre os dois comprimidos. Esses reconhecem que a CE, enquanto contraceptivo de emergência, não deve ser usado continuamente. $\mathrm{O}$ uso do método foi, utilizado de forma correta e não foi usado por todas as adolescentes que tiveram a sexarca ou alguma das demais relações sexuais sem proteção, o que pode representar falta de informação ou de acesso ao método pelo grupo.

Ressalta-se, o papel do enfermeiro como educador em saúde atuando em unidades básicas de saúde, entre outros espaços sociais, como a escola, devidamente capacitados para a função educativa, auxiliando o adolescente no processo de construção do conhecimento e sua utilização para o autocuidado.

\section{REFERÊNCIAS}

1. Amaral MA, Fonseca RMGS. Entre o desejo e o medo: as representações sociais das adolescentes acerca da iniciação sexual. Rev Esc Enferm USP. [Internet] 2006;40(4) [acesso em 30 ago 2010]. Disponível: http://www.scielo.br/scielo.php?script=sci_arttext\&pi $\mathrm{d}=$ S0080-62342006000400004

2. Saito MI, Leal MM. Adolescência e contracepção de emergência: fórum 2005. Rev. paul. pediatr. [Internet] 2007;25(2) [acesso em 22 mai 2012]. Disponível: http://www.scielo.br/scielo.php?pid=S010305822007000200014\&script $=$ sci_arttext

3. Vieira LM, Saes SO, Dória AAB, Goldberg TBL. Reflexões sobre a anticoncepção na adolescência no Brasil. Recife. Rev. Bras. Saude Matern. Infant. [Internet] 2006;6(1) [acesso em 30 ago 2010]. Disponível: http://www.scielo.br/scielo.php?pid=S151938292006000100016\&script $=$ sci_arttext

4. Madureira VSF, Trentini M. Da utilização do preservativo masculino à prevenção de DST/AIDS. Ciênc. saúde colet. [Internet] 2008;13(6) [acesso em 30 ago 2010]. Disponível:http://www.scielo.br/scielo.php?script=sci_ arttext\&pid=S1413-81232008000600015 
5. Teixeira AMFB, Knauth DR, Fachel JMG, Leal AF. Adolescentes e uso de preservativos: as escolhas dos jovens de três capitais brasileiras na iniciação e na última relação sexual. Cad. Saúde Pública. [Internet] 2006;22 [acesso em 30 ago 2010]. Disponível: http://www.scielo.br/scielo. php?script=sci_arttext\&pid=S0102-311X2006000700004

6. Souza RA, Brandão ER. Marcos normativo da anticoncepção de emergência e as dificuldades de sua institucionalização nos serviços públicos de saúde. Physis. [Internet] 2009;19(4) [acesso em 22 mai 2012]. Disponível: http://www.scielo.br/scielo.php?script=sci arttext\&pid=S0103-73312009000400009

7. Paiva SP, Brandão ER. Contracepção de emergência no contexto das farmácias: revisão crítica da literatura. Physis. [Internet] 2012;22(1) [acesso em 22 mai 2012]. Disponível: http://www.scielo.br/scielo.php?pid=S0103$73312012000100002 \&$ script $=$ sci arttext

8. Brasil. Brasília: Ministério da Saúde. Pesquisa Nacional de Demografia e Saúde da Criança e da Mulher de 2006. 2008.583 p.

9. Oliveira TC, Carvalho LP, Silva MA. O enfermeiro na atenção à saúde sexual e reprodutiva dos adolescentes. Rev Bras Enferm [Internet] 2008;61(3) [acesso em 30 ago 2010]. Disponível: http://www.scielo.br/scielo.php?pid=S003471672008000300005\&script=sci_arttext

10. Marques JF, Silva KM, Moreira KAP, Queiroz MVO. Saúde e cuidado na percepção de estudantes adolescentes: contribuições para a prática de enfermagem. Cogitare enferm. [Internet] 2012;17(1) [acesso em 18 mai 2012]. Disponível: http:/ojs.c3sl.ufpr. br/ojs2/index.php/cogitare/article/viewArticle/26372

11. Vieira RP, Machado MFAS, Bezerra IMP, Machado AS. Assistência à saúde e demanda dos serviços na estratégia saúde da família: a visão dos adolescentes. Cogitare enferm. [Internet] 2011;16(4) [acesso em 18 mai 2012]. Disponível: http://ojs.c3sl.ufpr.br/ojs2/index. $\mathrm{php} /$ cogitare/article/viewArticle/25443

12. Roman EP, Ribeiro RR, Guerra GJ, Barros AAF. Antropometria, maturação sexual e idade da menarca de acordo com o nível socioeconômico de meninas escolares de Cascavel. Rev. Assoc. Med. Bras. [Internet] 2009;55(3) [acesso em 22 mai 2012]. Disponível:http:// www.scielo.br/scielo.php?script=sci_arttext\&pid $=$ S0104-42302009000300026

13. Taquette SR. Atividade sexual de adolescentes femininas em contextos de pobreza. In: Taquette SR, organizador. Aids e juventude: gênero, classe e raça. Rio de Janeiro: EdUERJ; 2009. p. 55-66.
14. Araújo MSP, Costa LOBF. Comportamento sexual e contracepção de emergência entre adolescentes de escolas públicas de Pernambuco, Brasil. Cad. Saúde Pública. [Internet] 2009;25(3) [acesso em 22 mai 012]. Disponível: http://bases.bireme.br/cgi-bin/wxislind.exe/ iah/online/?IsisScript $=$ iah/iah.xis\&src $=$ google\&base $=$ LILACS\&lang $=$ p\&nextAction $=1 n k \&$ exprSearch $=5078$ $58 \&$ indexSearch $=$ ID

15. Jardim DP, Brêtas JRS. Orientação sexual na escola: a concepção dos professores de Jandira-SP. Rev Bras Enferm. [Internet] 2006;59(2) [acesso em 01 set 2010]. Disponível: http://www.scielo.br/scielo.php?pid=S0034$71672006000200007 \&$ script $=$ sci_arttext

16. Peretto M, Drehmer LBR, Bello HMR. O não comparecimento ao exame preventivo de câncer de colo uterino: razões declaradas e sentimentos envolvidos. Cogitare enferm. [Internet] 2012;17(1) [acesso em 18 mai 2012]. Disponível: http://ojs.c3sl.ufpr.br/ojs2/index. php/cogitare/article/viewArticle/26371

17. Almeida AH, Soares CB. Ensino de educação nos cursos de graduação em enfermagem. Rev Bras Enferm. [Internet] 2010;63(1) [acesso em 22 ma 2012]. Disponível:http:/www.scielo.br/scielo.php?pid=S0034$71672010000100018 \&$ script $=$ sci_arttext 